\title{
Disrupted cooperation between transcription factors across diverse cancer types
}

\author{
Jing Wang ${ }^{1+} \mathbb{D}$, Qi Liu ${ }^{1,2+}$, Jingchun Sun ${ }^{3}$ and Yu Shyr ${ }^{1,4,5^{*}}$
}

\begin{abstract}
Background: Transcription Factors (TFs), essential for many cellular processes, generally work coordinately to induce transcriptional change in response to internal and external signals. Disrupted cooperation between TFs, leading to dysregulation of target genes, contributes to the pathogenesis of many diseases, including cancer. Although the aberrant activation of individual TFs and the functional effects have been widely studied, the perturbation of TF cooperativity in cancer has rarely been explored.
\end{abstract}

Results: We used TF co-expression as proxy as cooperativity and performed a large-scale study on disrupted TF cooperation across seven cancer types. While the connectivity of downstream effectors, like metabolic genes and TF targets, were more or similarly disrupted than/with non-TFs, the cooperativity of TFs (upstream regulators) were consistently less disturbed in all studied cancer types. Highly coordinated TFs in normal, however, generally lost that cooperation in cancer. Although different types of cancer shared very few TF pairs with highly disrupted cooperation, the cooperativity of interferon regulatory factors (IRF) was highly disrupted in six cancer types. Specifically, the cooperativity of IRF8 was highly perturbed in lung cancer, which was further validated by two independent lung squamous cell carcinoma (LUSC) and lung adenocarcinoma (LUAD) datasets. More interestingly, the cooperativity of IRF8 was markedly associated with tumor progression and even contributed to the patient survival independent of tumor stage.

Conclusions: Our findings underscore the far more important role of TF cooperativity in tumorigenesis than previously appreciated. Disrupted cooperation of TFs provides potential clinical utility as prognostic markers for predicting the patient survival.

Keywords: Transcription factors, TF cooperation, Cooperation disruption, Pan-cancer, Co-expression

\section{Background}

Transcription factors (TFs) are proteins that bind to either promoter or enhancer regions of a gene, thereby regulating the transcription activity of the gene $[1,2]$. About $10 \%$ of genes in the human genome encode TFs and they are essential for many cellular processes [3]. A high proportion of TFs either acts as oncogenes or tumor suppressors or regulates the activity of pathways

\footnotetext{
* Correspondence: yu.shyr@vanderbilt.edu

${ }^{\dagger}$ Equal contributors

${ }^{1}$ Center for Quantitative Sciences, Vanderbilt University School of Medicine,

Nashville, TN, USA

${ }^{4}$ Department of Cancer Biology, Vanderbilt University School of Medicine,

Nashville, TN, USA

Full list of author information is available at the end of the article
}

related to tumor progression. The deregulation of these TFs, reshaping the expression of their target genes, leads to tumor formation, progression, and metastasis [4]. Numerous studies have revealed the functional impact of aberrant activation of individual TFs on tumor progression. For example, mutations of BRCA1 and BRCA2 cause the genetic instability of the cell and thus confer a substantial risk of breast and ovarian cancer [5].

Rather than function alone, transcription factors generally cooperate to control gene expression. Like aberrant activation of individual TFs, disruption of TF cooperativity also alters the expression of downstream genes and contributes to disease pathogenesis. Several studies have reported the alteration of TF cooperativity in cancer [6-15]. For example, the association between SNAIL and ZEB1 is 
lost in human colon carcinomas [16]. SNAIL, ZEB, and basic helix-loop-helix (bHLH) factors ally to mediate the dynamic silencing of $\mathrm{CDH} 1$, and loss of $\mathrm{CDH} 1$ function is a hallmark of carcinoma cell invasiveness $[17,18]$. Three transcription factors TTF1/NKX2-1, NKX2-8, and PAX9 show pronounced synergy in promoting the proliferation of immortalized human lung epithelial cells, and the alteration of their cooperativity contributes to lung cancer development [19]. Compared with the rapid accumulation of knowledge on individual TFs, however, there is only limited research on TF cooperativity and our understanding of TF cooperativity perturbation in cancer is lagging far behind.

Large-scale genomics projects, such as The Cancer Genome Atlas (TCGA), generating various omics data for thousands of tumors with matched normal samples [20], have provided a great opportunity to explore the common and specific disruption of gene co-expression across multiple cancer types. For example, West et al. has demonstrated that cancer is characterized by an increase in network entropy, i.e., reduced absolute gene correlations, while cell cycle/proliferation genes are preferentially associated with significant reductions in network entropy [21]. Here we focused on transcription factors, which are upstream regulators of transcriptome change that results in phenotypic change. We analyzed the dysregulated cooperation between TFs across seven cancer types and compared that with the dysregulation between non-TFs and other downstream effectors. We not only identified specific TF pairs highly disrupted in each individual cancer type but also detected common TFs whose cooperativity were significantly disturbed across diverse cancer types. Finally, the disrupted cooperation of IRF8 in lung cancer was validated by two independent lung squamous cell carcinoma (LUSC) and lung adenocarcinoma (LUAD) datasets, and its contribution to tumor progression and patient survival was further investigated.

\section{Results and discussion}

\section{TF cooperativity is less disturbed than non-TF in cancer}

We obtained 1991 human TFs in total by combining three databases, AnimalTFDB [22], TRANSFAC [23], and TFCat [24] (Methods). We studied seven cancer types and each cancer type had mRNA expression profiles measured for both tumor and matched normal samples (Table 1).

TF co-expression has been commonly used to predict TF cooperativity [25-27]. For instance, Hammonds et al. used TFs co-expression to suggest their co-association [25]. Zhou et al. developed a second-order expression similarity to infer TFs' cooperativity [27]. Here we used two lists of known cooperative TFs to study the relationship between co-expression and cooperativity. One included interacting TFs with high confidence from
Table 1 Sample size for each cancer type

\begin{tabular}{llll}
\hline & CancerType & Normal-tumor pair & Source \\
\hline Discovery & NSCLC & 85 & GSE32665 \\
& PRAD & 58 & GSE6919 \\
& COADREAD & 32 & GSE8671 \\
& BRCA & 61 & GSE14999 \\
& HNSC & 41 & TCGA \\
& KIRC & 72 & TCGA \\
& LIHC & 50 & TCGA \\
Validation & LUAD & 57 & TCGA \\
& LUSC & 50 & TCGA \\
\hline
\end{tabular}

HitPredict (http://hintdb.hgc.jp/htp/) [28] , and the other contained TF co-associations based on the non-randomly distributed TF binding regions from ENCODE [29]. We used Spearman correlation coefficient to measure the co-expression, which is more robust to outliers than Pearson correlation. Compared to random TF pairs, cooperative TFs were more tightly co-expressed in all the studied cancer types (Wilcoxon Rank Sum test, $p<1 \mathrm{e}-4$ ) (Additional file 1: Figure S1). Among 712 coassociated TFs in the HepG2 cell line, even higher correlations were observed in the corresponding liver cancer (LIHC) than general TF associations and random TF pairs (Additional file 1: Figure S1). These findings demonstrated that TF co-expression can act as an appropriate proxy for cooperativity.

There was no general bias for TFs on the measure of cooperativity compared to other genes. The scatterplot containing the pair-wise correlation coefficient on the yaxis and the average expression of genes ( $\log _{2}$ scale) on the $\mathrm{x}$-axis generally followed a horizontal line around zero in all tumor and normal samples (Additional file 2: Figure S2), suggesting no intensity-dependent bias on the measure of cooperativity. TFs had comparable concentrations with non-TFs in each individual cancer type and matched normal samples as well (Additional file 3: Figure S3). In addition, TFs, non-TFs and TF targets showed similar cooperativity level in all types of matched normal samples (Additional file 4: Figure S4).

To measure the alteration of cooperation, we used the absolute correlation change in tumor versus normal (Methods). Analysis of gene correlation change, i.e., differential co-expression analysis, is a more comprehensive technique to the differential expression analysis $[30,31]$. Correlation change between TFs is not only caused by expression alteration of individual TFs, but also driven by subtle perturbation in TFs expression coordination, which both potentially leads to the dynamic switch of TF partners [32, 33].

Although TF and non-TFs showed comparable cooperative level, the absolute correlation change between 
TFs was significantly smaller than that between non-TFs across all studied cancer types (Wilcoxon Rank Sum test, $p<2.2 \mathrm{e}-308$; difference between the medians (delta) $>0.01$ ) (Fig. 1). Specifically, breast invasive carcinoma (BRCA) exhibited the largest difference of correlation change in TFs compared to non-TFs, followed by kidney renal clear cell carcinoma (KIRC), liver hepatocellular carcinoma (LIHC), and non-small-cell lung carcinoma (NSCLC) (Fig. 1). In contrast, two other groups of genes, metabolic genes and TF target genes, did not show the consistent smaller correlation changes like what TFs presented across different cancer types. Instead, these two groups of genes either showed larger or same correlation change compared to those of non-TFs (Fig. 1). The fact that only the cooperation between TFs is significantly less disturbed than non-TFs in all types of tumor relative to normal tissues could be explained by the functional roles of transcription factors rather than statistical artifacts or other confounding factors. As upstream regulators for determining gene expression, the cooperativity between TFs is considerably constrained and more robust to disease than that of downstream effectors, like TF targets or genes in metabolic pathway [34]. Restricting our analysis on two lists of known cooperative TFs, however, we didn't observe the consistent constrained TF cooperativity across diverse cancer types, which was possibly due to the low coverage and bias of existing knowledge $[35,36]$. There are only 114 TFs with predicted associations from ENCODE, which covers only $5.7 \%$ of known TFs. Although there are much more TFs from HitPredict compared to ENCODE, they have similar number of TF interactions, which suggests the number of TF interactions of HitPredict is far less than expected. Moreover, protein-protein interaction networks are generally associated with multiple types of biases, e.g., selection preference (disease proteins) and technical limitations $[35,36]$.

\section{Tightly coordinated TFs lost cooperation in cancer}

Although the cooperativity between TFs is considerably constrained during tumorigenesis, some TF pairs significantly changed their coordination in cancer. We focused on TF pairs tightly coordinated in either normal or tumor samples (top $5 \%$ here). We tracked the cooperation changes of these TF pairs and classified them into three categories, "Gain", "Loss" and "Reverse" (Fig. 2a). TF pairs with significant coordination changes $(>2 \mathrm{SD})$ that only appeared in the highly coordinated list in tumor but not in normal were defined as "Gain". Accordingly, TF pairs with significant coordination changes $(>2 \mathrm{SD})$ that only presented in the highly coordinated normal list were defined as "Loss". TF pairs showing both in the tumor and normal list but opposite direction (e.g., positively correlated in tumor but inversely correlated in normal or vice versa) were classified as "Reverse". As expected, there were very few TF pairs belonging to the "Reverse" category across all studied cancer types. In most types of cancer, "Loss" is dominant over "Gain", especially in BRCA, NSCLC and KIRC (Fig. 2b). Due to the dominant effect of losing tightly coordinated TF pairs rather than gaining during tumorigenesis, highly coordinated TF pairs in tumor were less disturbed than those in normal. In BRCA, the highly cooperative TFs in tumor were even less altered than other TFs (Additional file 5: Figure S5). Consistent with previous studies revealing cancer is globally characterized by reduced absolute gene correlations [21], our results indicate that tightly coordinated TFs losing their normal cooperation play a major role in tumorigenesis. That is, disrupting the normally coordinated TFs affect gene

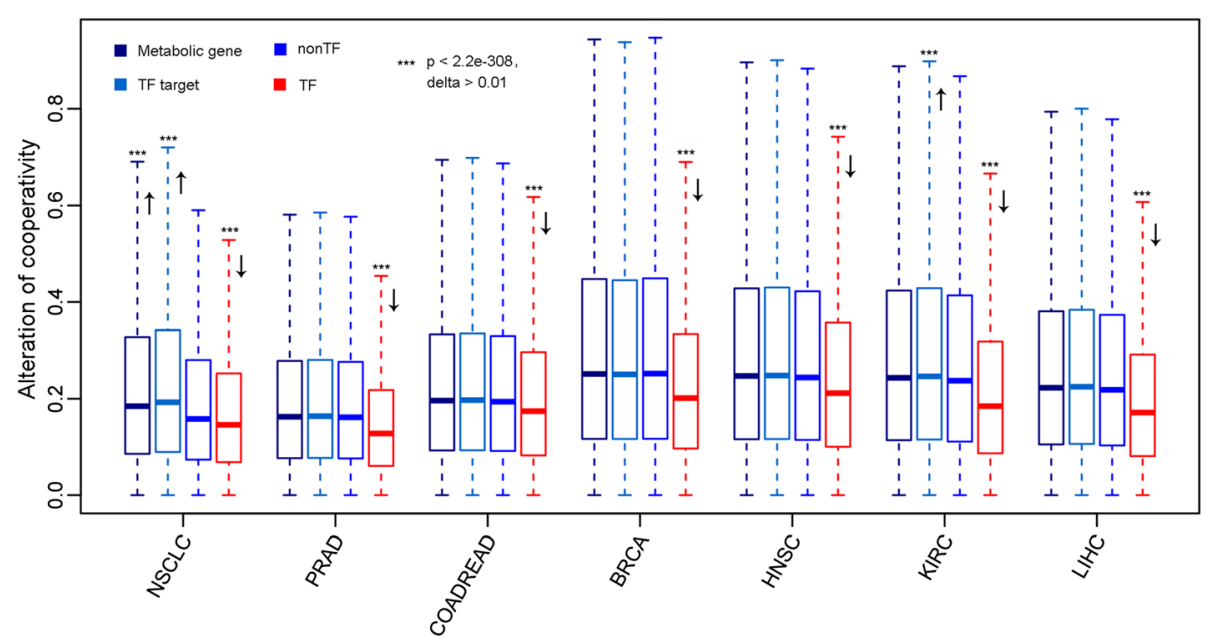

Fig. 1 Cooperativity alterations in tumor versus normal. The box plots illustrate the absolute correlation change in tumor relative to normal between TF pairs (red), non-TF pairs (blue), metabolic genes (steel blue) and TF target genes (light blue) in NSCLC, PRAD, COADREAD, BRCA, HNSC, KIRC, and LIHC 


\section{a}

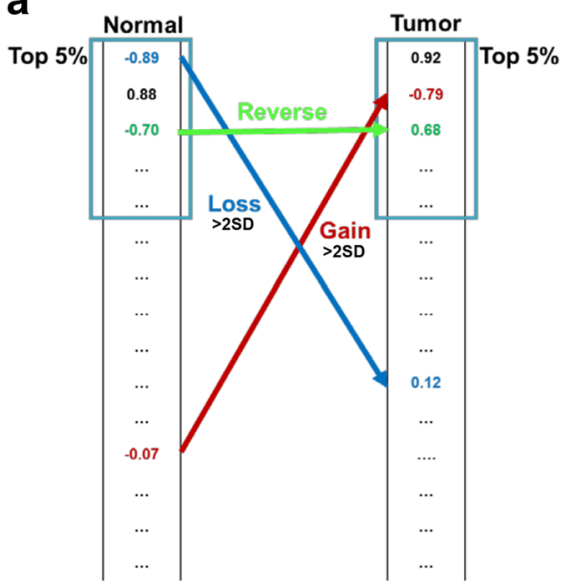

b

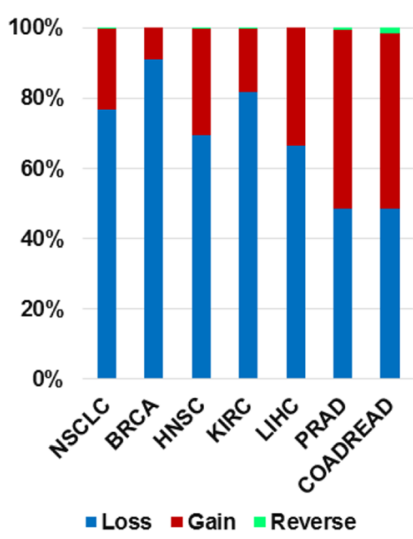

Fig. 2 The pair-wise TF cooperativity is mainly lost in tumor relative in normal. a The schema of TF cooperation change. TF pairs with significant coordination changes (>2SD) that only appeared in the highly coordinated list in tumor but not in normal were defined as "Gain". TF pairs with significant coordination changes ( $>2$ SD) that only presented in the highly coordinated normal list were defined as "Loss". TF pairs showing both in the tumor and normal list but opposite direction (e.g., positively correlated in tumor but inversely correlated in normal or vice versa) were classified as "Reverse". b Bar plot depicts percentage of Loss, Gain and Reverse in TF cooperativity alteration

expression of their target genes, leading to tumor initiation and progression.

\section{Highly dyscooperated TFs across diverse cancer types}

We ranked the disrupted TF pairs in each type of cancer, trying to identify the TF pairs whose cooperativity is commonly disturbed across diverse cancer types. We found different cancer types shared very few highly disrupted TF pairs. Among the top 3000 TF pairs with the highest disruption in cooperativity, there were only $5 \sim$ 16 TF pairs shared between two cancer types (Fig. 3a). Moreover, there were only 5 TF pairs shared by three or
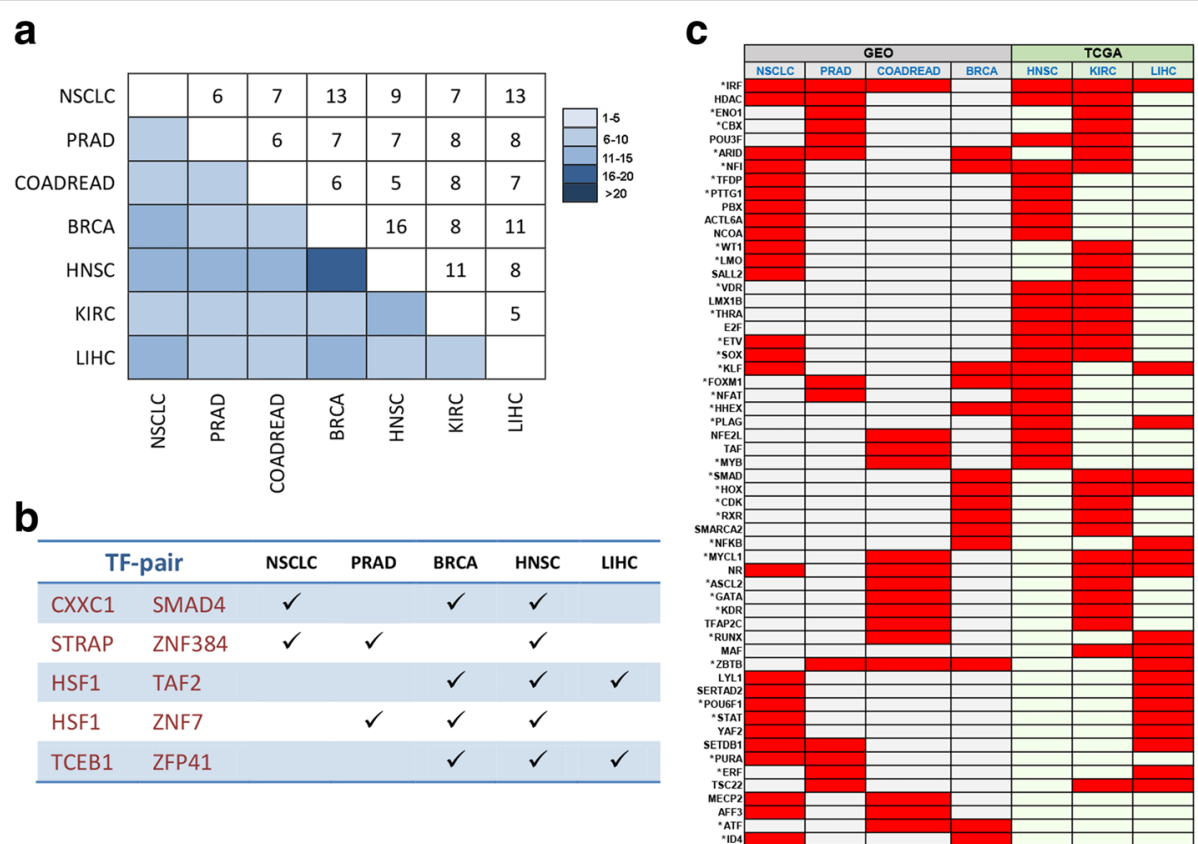

Fig. 3 Highly disrupted TFs across diverse cancer types. a Heat map of highly disrupted TF pairs shared across different cancer types. Upper triangle shows the number of common pairs. $\mathbf{b}$ A list of highly disrupted TF pairs shared by more than two cancer types. $\mathbf{a}$ and $\mathbf{b}$ are based on the top 3000 TF pairs with the highest disruption in cooperativity. c Heat map of common highly disrupted TFs across different cancer types. Red bars designate that the TF labeled on the left is identified in the cancer type labeled on the top with greatly disturbed cooperativity with other TFs. Datasets from GSE and TCGA are shaded in different colors. TFs marked with "** function association with tumor progression 
more cancer types (Fig. 3b). The correlation plots of these $5 \mathrm{TF}$ pairs and correlation measures in normal and tumor were given in Additional file 6: Figure S6. Consistent with our previous finding, most of TF pairs lose cooperation during tumorigenesis, such as CXXC1SMAD4 in NSCLC and BRCA. We also observed that few uncorrelated TFs in normal became co-expressed in tumor, e.g., HSF1-ZNF7 in BRCA and HNSC (Additional file 6: Figure S6), indicating abnormal co-expression potentially drive tumorigenesis as well. TAF2 and HSF1 were identified as driver genes of liver carcinoma and closely linked on chromosome 8q24 [37, 38]. This finding reveals that tumor is quite different from each other on disrupted TF pairs. Among the top 3000 TF pairs with the highest disruption in cooperativity in each cancer type, less than $50 \%$ of TF pairs were due to the differential expression of TF itself except KIRC (62.3\%). Among the 5 common TF pairs, only the disruption of TCEB1-ZFP41 in LIHC was caused by the differential expression of ZFP41 $\left(\left|\log _{2} \mathrm{FC}\right|>1\right.$ \& FDR $\left.<0.05\right)$.

We aggregated the disrupted cooperation of TF pairs to each individual TF by calculating the number of significantly changed cooperation for this TF (Methods). The TFs with significant number of broken cooperative relationship are called "highly dyscooperated" TFs. As a result, more than 20 highly dyscooperated TFs were identified for each cancer type. KIRC identified 87 highly dyscooperated TFs, and NSCLC discovered 61, and 44, 42, 34, 28 and 24 were recognized for LIHC, HNSC, COADREAD, PRAD, and BRCA respectively (Additional file 7: Table S1). Less than $50 \%$ of these dyscooperated TFs were differentially expressed in the corresponding cancer type $\left(\left|\log _{2} \mathrm{FC}\right|>1\right.$ \& FDR $\left.<0.05\right)$. Compared with the low overlap of disrupted TF pairs, the dyscooperated TFs were much more common between different types of cancer, which either share the same TFs or TFs belonging to the same family. For example, NSCLC shared $47.5 \%$ of its highly dyscooperated TFs/TF families with other cancer types (Fig. 3c). Notably, there were 57 TFs/TF families common in two or more cancer types (Fig. 3c). These highly disrupted TF families included well-known genes related to tumor progression, such as the HOX family, the $Z B T B$ family, WT1, and so on [39-41]. Most interestingly, the interferon regulatory factors (IRF) family was detected in six out of the seven studied cancers. Specifically, IRF8 was identified as one of the most dyscooperated TFs in NSCLC and COADREAD, IRF6 was detected in PRAD, IRF5 was found in HNSC and KIRC, and IRF2 was discovered in LIHC (Fig. 3c). IRF family members share a DNAbinding domain (DBD) and recognize a consensus motif, 5'-AANNGAAA-3' [42]. Previous studies have revealed the versatile and critical functions performed by the IRF family transcription factors, including immune response, cell growth regulation, cell apoptosis, and hematopoietic development [43-45]. Moreover, IRF family members contribute to tumorigenesis and tumor progression. For example, IRF6 interacts with maspin (mammary scrine protease inhibitor), which is characterized as a tumor suppressor [46]. The targets of IRF8 are involved in antiapoptotic function [47]. Reported as a direct target of $p 53$ [48], IRF5 can also regulate the cell cycle and apoptosis in a $p 53$-independent way $[49,50]$. IRF2 is an oncogenic gene which plays a positive role in the cell cycle regulation of the human histone H4 gene FO108 [51]. Taken together, it can be inferred that the dyscooperated IRF family might be a common mechanism in tumor initiation or progression.

\section{Functional effect of dyscooperated IRF in tumorigenesis}

GO enrichment analysis was performed for the target genes of IRF transcription factors, which were identified by motif searching and further filtered by chromatin accessibility from DNase-seq data in the corresponding cell lines, to investigate their potential functional roles. The target genes are enriched in many cancer related functions such as cell death, response to stress, cell proliferation, and regulation of apoptotic processes. Furthermore, 132 (accounting for $25.3 \%$ ) genes are annotated as cancer census genes in the Cancer Gene Census of COSMIC [52]. Among them, BRAF is an important gene associated with various cancers, including colorectal cancer, malignant melanoma, thyroid carcinoma, nonsmall cell lung carcinoma, and adenocarcinoma of lung [53, 54]; NDRG1 acts as a tumor suppressor gene involved in stress responses, cell growth, and differentiation, and its expression may be a prognostic indicator for several types of cancer [55-57].

We explored the potential cause and functional effect of dyscooperated IRF family transcription factors in the six cancers. We first compared the expression abundance of IRF transcription factors in tumor versus normal to see whether the dyscooperation is caused by differential expression of the TF itself. We found that the IRF transcription factor was not significantly differentially expressed in each tumor versus the matched normal (Fig. 4), which means the dyscooperation of IRF members was caused by perturbation in expression coordination rather than expression alteration of the IRF itself. Moreover, the expression abundances of IRF target genes were more significantly disturbed than non-target genes (Kolmogorov-Smirnov test, the $p$-value in NSCLC, PRAD, COADREAD, HNSC, KIRC and LIHC is 0, 5.66e-4, $1.51 \mathrm{e}-9,9.06 \mathrm{e}-7,3.67 \mathrm{e}-10$ and $4.35 \mathrm{e}-6$ respectively) (Fig. 5). To reduce the potential bias introduced by the global effect of tumorigenesis, we further compared the expression alteration of IRF's targets and non-targets involved in the stress response. As a result, the targets involved in stress response were more likely to be dysregulated than non-targets 

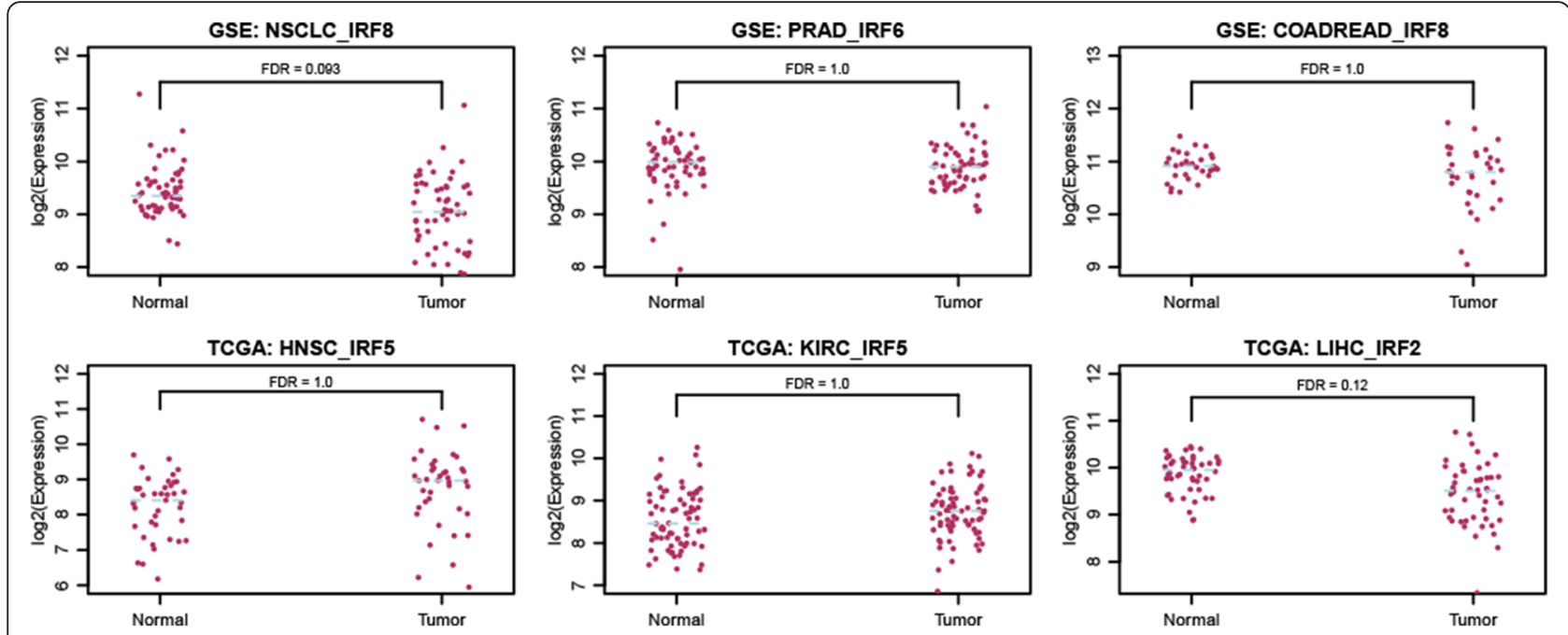

Fig. 4 Expression abundance of IRF in tumor versus normal. Each point in the plot represents a sample. FDR is given on the top, and the median concentration in tumor/normal is shown by a dashed line

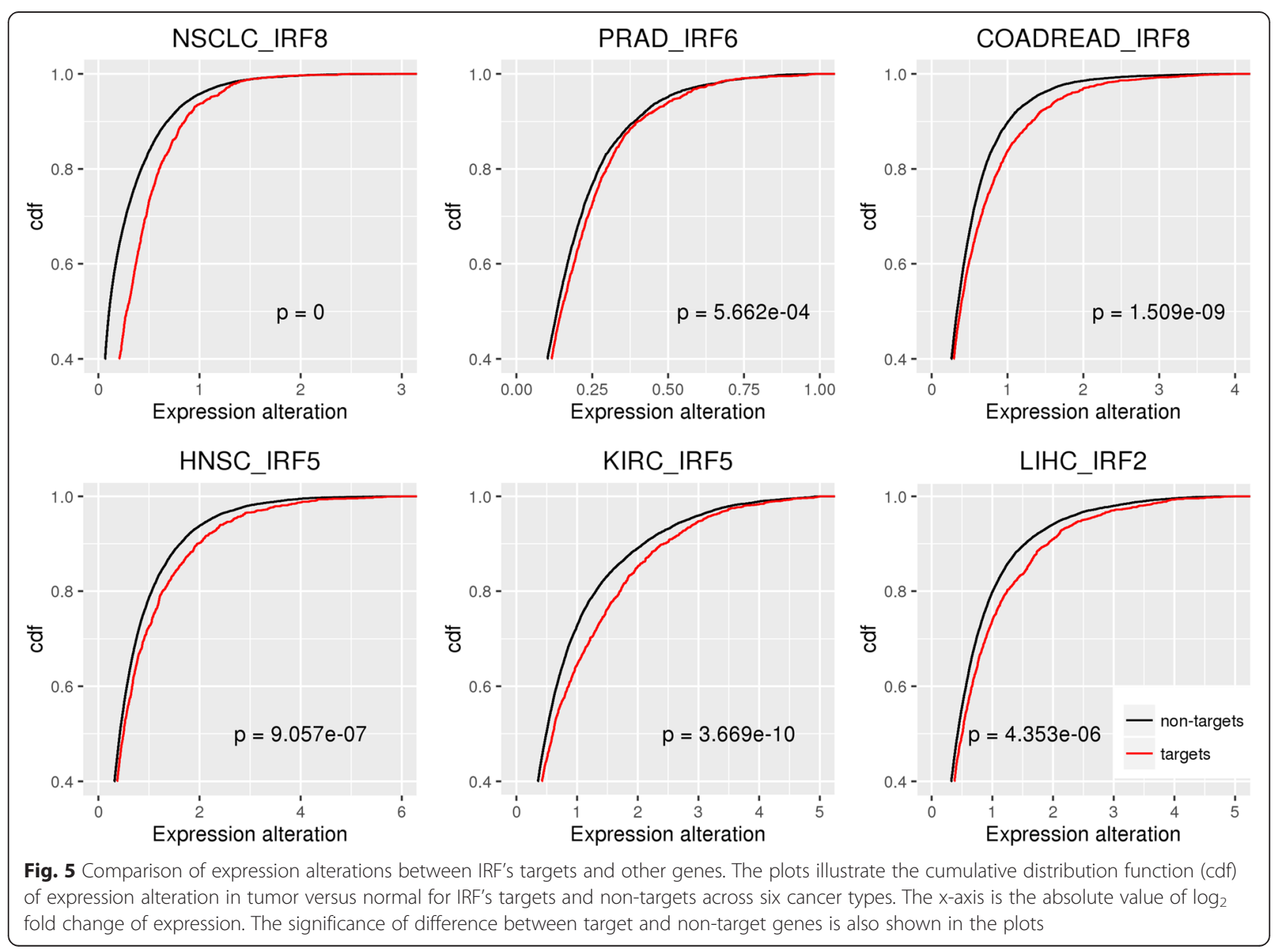


with the same function across all six cancer types (Additional file 8: Figure S7). These results demonstrated that disturbance of the IRF cooperativity affects the transcription of downstream target genes. The observation that the dysregulation of the target genes is mainly caused by the disrupted TF cooperation rather than differentially expressed TF itself suggests that the analysis of TF cooperativity in tumor is more important than previously recognized. It holds great potential for identifying novel drivers that could never be discovered by regular differential expression analysis.

\section{Validation of dyscooperated IRF8 using LUAD and LUSC datasets}

We downloaded two public RNA-seq datasets from TCGA and performed the same analysis on the disrupted TF cooperation. One includes 57 LUAD patient tumor and adjacent normal samples, and the other consists of 50 LUSC tumor and matched normal samples (Table 1). Consistent with our observation in the discovery cohort, we found TFs had comparable expression abundances with non-TFs in both LUAD and LUSC datasets. Highly coordinated TF pairs dominantly lost cooperativity during tumorigenesis, and those highly coordinated TF pairs in tumor were less disturbed than those in normal (Fig. 6a). We further identified dyscooperated TFs in these two datasets. The results showed that 13 out of the 61 identified highly dyscooperated TFs in the discovery NSCLC cohort were confirmed in the LUSC and/or LUAD datasets
(Fig. 6b). The IRF family member, IRF8, was confirmed in both LUAD and LUSC datasets. Moreover, seven additional highly dyscooperated TFs were detected in both LUAD and LUSC (Fig. 6b). Additionally, the expression of IRF8's targets was more significantly altered than nontargets in tumor versus normal (Kolmogorov-Smirnov test, p-value $<3.16 \mathrm{e}-14$, Fig. 6c). Repeated studies on the two independent datasets reproduced the findings from the discovery cohort, which demonstrate that the disruption of TF cooperativity is highly reproducible and biological meaningful in cancer.

Tumors progress through a series of stages, which is the most important factor in prognosis. We divided the LUSC patients into different groups based on tumor stages and aggregated the cooperativity of IRF8 in each individual group. We combined stage III and IV together since there are only few samples in stage IV. As a result, we have 211 stage I, 123 stage II, and 82 stage III/IV samples, respectively. Notably, we found that the cooperativity of IRF8 was remarkably inversely correlated with tumor stages. As the tumor stage increasing, IRF8 cooperation with other TFs became looser and looser (Fig. 7a), which indicates that IRF8 cooperativity might play an important role in cancer development. More importantly, we investigated the survival contributions of IRF8 cooperativity within each LUSC stage. We divided patients into two groups according to survival time, long-survival and short-survival. We found that IRF8 was more tightly cooperated with other TFs in patients a
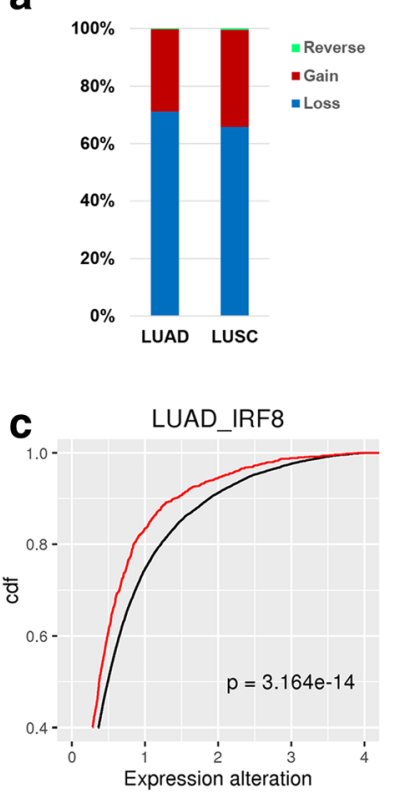
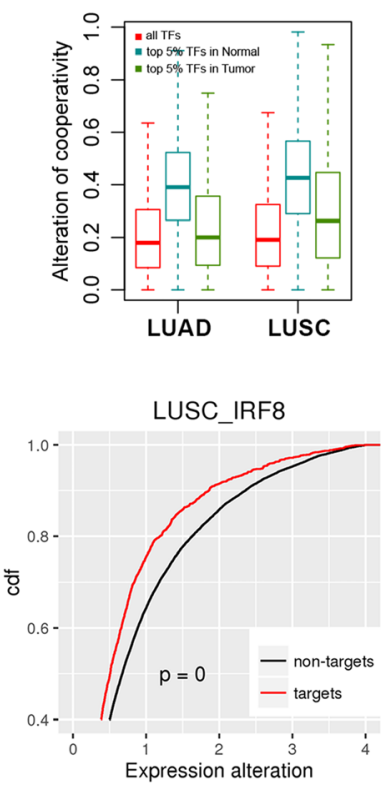

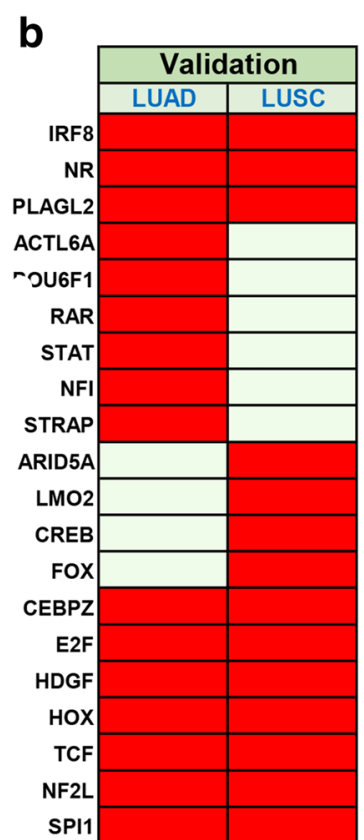

Fig. 6 Validation of dyscooperated IRF8 in LUAD and LUSC. a Cooperativity alterations between TF pairs that highly coordinated in normal/ tumor. b Highly dyscooperated TFs also identified in the previous NSCLC dataset. c Comparison of expression alteration between IRF8's target and non-target genes 

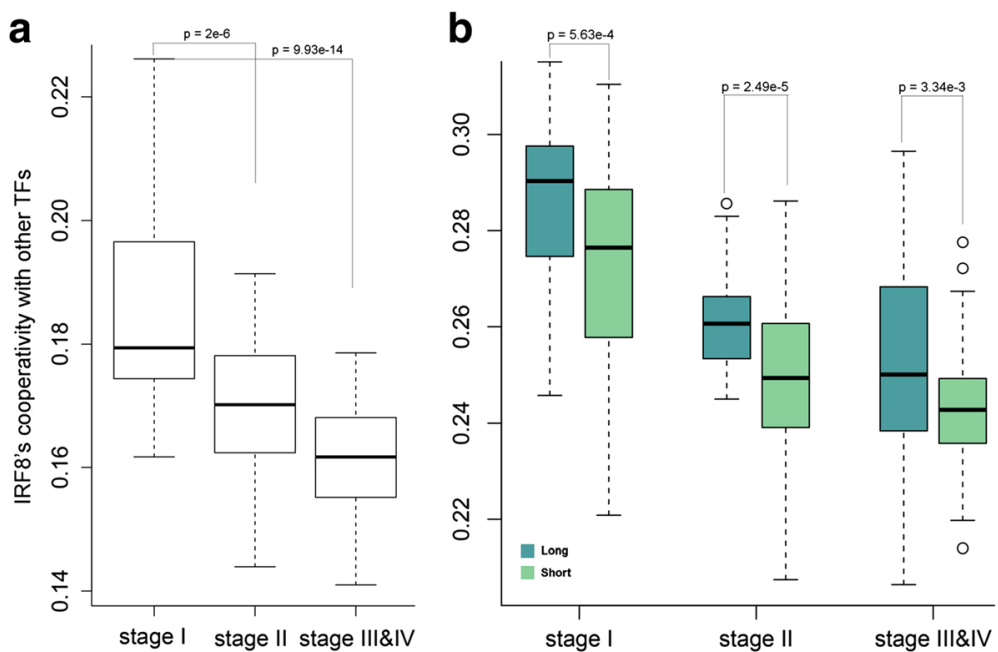

Fig. 7 The IRF8 cooperativity associated with tumor progression and patient survival. a IRF8 loosens its cooperation with other TFs along with cancer progression in LUSC. $\mathbf{b}$ IRF8 is more loosely coordinated with other TFs in patients with poor survival within each LUSC stage

with long survival than those with short survival within the same tumor stage (Fig. 7b, Wilcoxon Rank Sum test, the p-value is $5.63 \mathrm{e}-4,2.49 \mathrm{e}-5$, and $3.34 \mathrm{e}-3$ for stage I, II, and III\&IV patients respectively). That is, the cooperativity of IRF8 contributes to the survival of patients independent of tumor stage. These results demonstrated that the disruption of TF cooperation provides clinical utility for predicting patient survival.

\section{Conclusions}

We characterized the disrupted cooperation of transcription factors in cancer and identified common TFs whose cooperativity was highly disrupted across diverse cancer types. Our findings underscore the far more important role of TF cooperativity in tumorigenesis than previously recognized. Disrupted cooperation of TFs provides potential clinical utility as prognostic markers for predicting the patient survival.

\section{Methods}

\section{Datasets}

The gene expression profile analysed in this study included both next-generation sequencing (NGS) and microarray data. For the seven cancer types for discovery analysis, the mRNA-seq data of HNSC, KIRC, and LIHC were downloaded from Firehose developed by the Broad GDAC (https://confluence.broadinstitute.org/display/GDAC/Dashboard-Stddata). The microarray data for NSCLC (GSE19804), PRAD (GSE6919), COADREAD (GSE8671), and BRCA (GSE14999) were downloaded from the Gene Expression Omnibus (GEO, http:// www.ncbi.nlm.nih.gov/geo/) [58]. The two independent datasets for validation, LUAD and LUSC, and the clinical data were also obtained from Firehose. The detailed information about the sample size and source for each dataset is shown in Table 1. We excluded genes if they were not expressed in more than $50 \%$ of samples for TCGA RNA-seq data. For affymetrix microarray data, we applied data-dependent cutoffs to remove lowly expressed genes based on their average signal $\left(\log _{2}\right.$ signal intensity $<7$ for GSE32665, $\log _{2}$ signal intensity <2 for GSE6919 and GSE8671).

After combining 1469 TFs in AnimalTFDB (v2.0) [22], 1837 in TRANSFAC (v2014.1) and 405 in TFCat [23, 24] and removing redundant TFs, we obtained 1991 human TFs in total (Additional file 9: Figure S8). The metabolic genes were downloaded from UniProt-GOA (release 2014_11, GO: 0008152) [59], and the TF target genes were downloaded from MSigDB (v5.0, C3: motif gene sets, TFT: transcription factor targets) [60].

\section{Disruption of cooperativity between genes}

We used co-expression as proxy for cooperativity. Spearman correlation coefficients were used to measure the correlation between any pair of genes, which is more robust to outliers than Pearson correlation. The absolute correlation change of any pair of genes in tumor relative to normal was used to measure the alteration of cooperativity. The cooperativity alteration between gene $i$ and $j$ was defined as:

$$
\left|C_{i j}^{T-N}\right|=\left|C_{i j}^{T}-C_{i j}^{N}\right|
$$

where $C_{i j}^{T}$ is the correlation between gene $i$ and $j$ in tumor, and $C_{i j}^{N}$ denotes the correlation in matched normal tissue. We compared the cooperativity alteration between TF pairs $(i \in T F \mathcal{E} j \in T F, \mathrm{j} \neq i)$, gene pairs in metabolic pathway, and TF target pairs with non-TF 
pairs $(i \notin T F \mathcal{E} j \notin T F)$ using the Wilcoxon Rank Sum test [61]. We used the criteria that the median difference is greater than 0.01 and the p-value is less than $1 \mathrm{e}-20$.

To assess the disruption of the cooperation of individual TF $i$ in cancer $C_{i}^{T-N}$, we aggregated the cooperativity alterations of this TF with other TFs. The cooperativity alteration between TF $i$ and other TFs $j(j \neq i, j \in T F)$ was $z$-transformed and the number of significantly changed cooperation, positive change or negative changes, was counted separately,

$$
C_{i}^{T-N} \rightarrow \begin{cases}\sum_{j=1, j \neq i}^{n} P_{i j}, & \text { if } Z_{C_{i j}^{T-N}}>3 P_{i j}=1 ; \text { otherwise } P_{i j}=0 \\ \sum_{j=1, j \neq i}^{n} N_{i j}, & \text { if } Z_{C_{i j}^{T-N}}<-3 N_{i j}=1 ; \text { otherwise } N_{i j}=0\end{cases}
$$

where $n$ is the number of TFs, and $Z_{C_{i j}^{T-N}}$ is the z-score of the cooperation change between TF $i$ and $j$ in tumor versus normal. The change is considered significant if the $\mathrm{z}$-score is greater than 3 or less than -3 . We identified highly disrupted TFs by setting the cutoffs. The cutoffs were chosen where the decreasing number of highly disrupted TFs was less than or equal to 10 as the increasing requirement of the number of significantly changed cooperation (Additional file 10: Figure S9).

\section{Statistical analysis}

The gene expression abundances were $\log _{2}$ transformed and the paired $\mathrm{t}$-test was used to identify differentially expressed genes between tumor and matched normal tissues. The Kolmogorov-Smirnov test was used to compare the expression changes between IRF's targets and non-targets, and between IRF's targets and nontargets involved in stress response. The Wilcoxon Rank Sum test was applied to compare the cooperativity alteration of TF pairs, metabolic gene pairs, and TF target pairs with that of non-TF pairs. The BH method was used to adjust p-values for multiple testing. All statistical tests in this study were implemented by $\mathrm{R}$ (version 3.0.3).

\section{IRF targets identification and $\mathrm{GO}$ enrichment analysis}

Match program (version 1.0) [62], provided by TRANSFAC database, was used to identify putative IRF binding sites in the promoter regions, which were defined as $500 \mathrm{bp}$ upstream and 100bp downstream of the transcription start site. The characteristic motifs for binding sites searching were obtained from TRANSFAC and listed in Table 2. Motif hits were further filtered by only considering those that fall in chromatin accessible regions for each type of cancer, which were obtained from ENCODE DNase-seq data (Additional file 11: Table S2). For example, we identified 1546 IRF8's targets in NSCLC
Table 2 Motifs for binding sites searching

\begin{tabular}{llllll}
\hline Motif & Matrix_acc $^{\mathrm{a}}$ & IRF2 & IRF5 & IRF6 & IRF8 \\
\hline 5'-RAAANTGAAAN-3' & M00972 & $\checkmark$ & $\checkmark$ & & $\checkmark$ \\
5'-BNCRSTTTCANTTYY-3' & M00772 & $\checkmark$ & $\checkmark$ & $\checkmark$ & $\checkmark$ \\
5'-GAAAAGYGAAASY-3' & M00063 & $\checkmark$ & & & \\
5'-RAARTGAAACTG-3' & M00699 & & & & $\checkmark$ \\
\hline
\end{tabular}

a Matrix_acc means the accession number of matrix in TRANSFAC

and 431 of them are involved in the function of stress response (Additional file 12: Table S3). The GO enrichment analysis for IRFs targets was performed via WebGestalt [63]. Functional categories with FDR $<0.0001$ were reported.

The cooperation of IRF8 associated with clinical outcome We studied the relationship between the IRF8 cooperativity and clinical outcome on LUSC dataset, which provided relatively more complete clinical information than the LUAD dataset. In total, 416 LUSC patients were included, among which, 211 were classified as stage I, 123 as stage II, and 82 as stage III/IV. We quantified the cooperativity of IRF8 by summarizing its cooperation score with other TFs,

$$
C_{I R F 8}=\sum_{j=1, T F_{j} \neq I R F 8}^{n} C_{I R F 8, j}
$$

where $C_{I R F 8, j}$ denotes the cooperation of IRF8 with TF $j$, and $\mathrm{n}$ is the number of TFs. To make the cooperativity of IRF8 comparable between stages, we applied the subsampling technique. We selected $80 \%$ of 82 patients (the smallest sample size of LUSC stages) and calculated the cooperativity of IRF8 in each resampling dataset, and we ran 50 resamplings. We compared the distribution of cooperativity of IRF8 between different stages and found that IRF8 has the highest cooperativity in stage I but the lowest cooperativity in stage III/IV.

To further investigate the contribution of IRF8 cooperativity to patient survival independent of tumor stage, we classified patients into two groups by their survival time: long-survival (top $25 \%$ ) and short-survival (bottom $25 \%$ ) within each stage. We compared the IRF8's cooperativity between these two groups.

\section{Additional files}

Additional file 1: Figure S1. The correlation between TF pairs from different datasets. The box plots illustrate the correlation (absolute value of Spearman correlation coefficient) between interacted TFs from HitPredict (HitPredict-TF), associated TFs and HepG2-specific associated TFs from ENCODE (ENCODE associated-TF), and the correlation of random TF pairs (random TF) in NSCLC, PRAD, COADREAD, BRCA, HNSC, KIRC, and LIHC. (TIF $189 \mathrm{~kb})$ 
Additional file 2: Figure S2. The impact of gene expression abundance on the measure of cooperation. The scatterplots containing the pair-wise correlation coefficient on the $y$-axis and the average expression of genes ( $\log _{2}$ scale) on the $x$-axis. (TIF $316 \mathrm{~kb}$ )

Additional file 3: Figure S3. Transcriptional abundances of TFs and non-TFs across cancers. TFs show comparable expression abundance with non-TFs in both normal and tumor. TFs are colored in red and non-TFs are colored in blue respectively. The expression value is $\log _{2}$ transformed. (TIF $193 \mathrm{~kb}$ )

Additional file 4: Figure S4. Cooperation of TFs, non-TFs, TF-targets and metabolic genes in normal samples. TFs, non-TFs and TF targets showed similar cooperativity level in all types of matched normal samples. (TIF $181 \mathrm{~kb}$ )

Additional file 5: Figure S5. Comparison of cooperation alterations between highly coordinated TFs in normal and those in tumor. (TIF $153 \mathrm{~kb}$ )

Additional file 6: Figure S6. The correlation measures of recurrent TF pairs in normal and tumor. Normal samples are colored in dark blue while the tumor ones are colored in dark red. $r_{\mathrm{N}}$ and $r_{T}$ denote the Spearman correlation coefficients in normal and tumor respectively. (TIF $1020 \mathrm{~kb}$ )

Additional file 7: Table S1. Identified highly dyscooperated TFs of each cancer type. (XLSX $15 \mathrm{~kb}$ )

Additional file 8: Figure S7. Comparison of expression alterations of IRF's targets and other genes involved in RS. Each of the six plots illustrates the cumulative distribution function (cdf) of expression change in tumor relative to normal for IRF's targets versus other genes involved in the GO term of response to stress (RS). The $x$-axis is the absolute value of $\log _{2}$ transformation of fold change. (TIF $16347 \mathrm{~kb}$ )

Additional file 9: Figure S8. The source of TFs data sets. (TIF $112 \mathrm{~kb}$ )

Additional file 10: Figure S9. Cut-offs for identifying highly dyscooperated TFs. (A) The distribution of the number the Z-transformed cooperativity alteration $\left(Z_{C_{i j}^{T-N}}\right)$ greater than 3 . (B) The distribution of the number the Z-transformed cooperativity alteration $\left(Z_{C_{i-N}^{T-N}}\right)$ less than -3 . The cut-off for identifying highly disrupted TFs is shown by the vertical line for each cancer type. (TIF $23144 \mathrm{~kb}$ )

Additional file 11: Table S2. Encode DNase-seq data used to filter motif hits. (XLSX $10 \mathrm{~kb}$ )

Additional file 12: Table S3. List of IRF8's targets and those involved in stress response in NSCLC. (XLSX $32 \mathrm{~kb})$

\section{Abbreviations}

bHLH, basic helix-loop-helix; BRCA, breast invasive carcinoma; COADREAD, colorectal adenocarcinoma; GEO, Gene Expression Omnibus; HNSC, head and neck squamous cell carcinoma; IRF, interferon regulatory factor; KIRC, kidney renal clear cell carcinoma; LIHC, liver hepatocellular carcinoma; LUAD, lung adenocarcinoma; LUSC, lung squamous cell carcinoma; NGS, next-generation sequencing; NSCLC, non-small-cell lung carcinoma; PRAD, prostate adenocarcinoma; RS, response to stress; TCGA, The Cancer Genome Atlas; TF, transcription factor.

\section{Acknowledgements}

We would like to thank Margot Bjoring and Page Hoskins for editorial support. We thank the anonymous reviewers who helped to enhance the quality of this paper.

\section{Funding}

This work was supported by National Cancer Institute (5U01 CA163056-05 to YS), Cancer Center Support Grant (2P30 CA068485-19 to YS), and the Robert J. Kleberg, Jr. and Helen C. Kleberg Foundation (CQS Pilot Project FY2016 to QL).

\section{Availability of supporting data}

The data sets supporting the results of this article are included within the article and its additional files.

\section{Authors' contributions}

JW carried out the analysis and drafted the manuscript. QL conceived of the study, guided the analysis, and revised the manuscript. JS helped in data analysis. YS supervised the research. All authors read and approved the final manuscript.

\section{Competing interests}

The authors declare that they have no competing interests.

\section{Consent for publication}

Consent for publication is not applicable in this study, because there are not any individual person's data.

Ethics approval and consent to participate

Not applicable.

\section{Author details}

${ }^{1}$ Center for Quantitative Sciences, Vanderbilt University School of Medicine, Nashville, TN, USA. ${ }^{2}$ Department of Biomedical Informatics, Vanderbilt University School of Medicine, Nashville, TN, USA. ${ }^{3}$ School of Biomedical Informatics, The University of Texas Health Science Center at Houston, Houston, TX, USA. ${ }^{4}$ Department of Cancer Biology, Vanderbilt University School of Medicine, Nashville, TN, USA. ${ }^{5}$ Department of Biostatistics, Vanderbilt University School of Medicine, Nashville, TN, USA.

Received: 20 November 2015 Accepted: 15 June 2016

Published online: 05 August 2016

\section{References}

1. Mitchell PJ, Tjian R. Transcriptional regulation in mammalian cells by sequence-specific DNA binding proteins. Science. 1989;245(4916):371-8.

2. Ptashne M, Gann A. Transcriptional activation by recruitment. Nature. 1997;386(6625):569-77.

3. Babu MM, Luscombe NM, Aravind L, Gerstein M, Teichmann SA. Structure and evolution of transcriptional regulatory networks. Curr Opin Struct Biol. 2004;14(3):283-91.

4. Libermann TA, Zerbini LF. Targeting transcription factors for cancer gene therapy. Curr Gene Ther. 2006;6(1):17-33.

5. Antoniou A, Pharoah PD, Narod S, Risch HA, Eyfjord JE, Hopper JL, Loman N

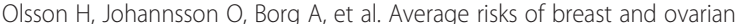
cancer associated with BRCA1 or BRCA2 mutations detected in case Series unselected for family history: a combined analysis of 22 studies. Am J Hum Genet. 2003;72(5):1117-30

6. Kerppola TK, Curran T. Fos-Jun heterodimers and Jun homodimers bend DNA in opposite orientations: implications for transcription factor cooperativity. Cell. 1991;66(2):317-26.

7. Mirny LA. Nucleosome-mediated cooperativity between transcription factors. Proc Natl Acad Sci U S A. 2010;107(52):22534-9.

8. Glasmacher E, Agrawal S, Chang AB, Murphy TL, Zeng W, Vander Lugt B, Khan AA, Ciofani M, Spooner CJ, Rutz S, et al. A genomic regulatory element that directs assembly and function of immune-specific AP-1-IRF complexes. Science. 2012;338(6109):975-80.

9. Li P, Spolski R, Liao W, Wang L, Murphy TL, Murphy KM, Leonard WJ. BATF-JUN is critical for IRF4-mediated transcription in T cells. Nature. 2012:490(7421):543-6.

10. Tussiwand R, Lee WL, Murphy TL, Mashayekhi M, KC W, Albring JC, Satpathy AT, Rotondo JA, Edelson BT, Kretzer NM, et al. Compensatory dendritic cell development mediated by BATF-IRF interactions. Nature. 2012;490(7421):502-7.

11. Ciofani M, Madar A, Galan C, Sellars M, Mace K, Pauli F, Agarwal A, Huang W, Parkurst CN, Muratet $M$, et al. A validated regulatory network for Th17 cell specification. Cell. 2012;151(2):289-303.

12. Medici D, Hay ED, Goodenough DA. Cooperation between snail and LEFtranscription factors is essential for TGF-beta1-induced epithelialmesenchymal transition. Mol Biol Cell. 2006;17(4):1871-9.

13. Sakai D, Suzuki T, Osumi N, Wakamatsu Y. Cooperative action of Sox9, Snail2 and PKA signaling in early neural crest development. Development. 2006;133(7):1323-33.

14. Zhang Y, Feng XH, Derynck R. Smad3 and Smad4 cooperate with c-Jun/cFos to mediate TGF-beta-induced transcription. Nature. 1998;394(6696):909-13.

15. Chen J, Yusuf I, Andersen HM, Fruman DA. FOXO transcription factors cooperate with delta EF1 to activate growth suppressive genes in B Iymphocytes. J Immunol. 2006;176(5):2711-21.

16. Pena C, Garcia JM, Garcia V, Silva J, Dominguez G, Rodriguez R, Maximiano C, Garcia de Herreros A, Munoz A, Bonilla F. The expression levels of the transcriptional regulators p300 and CtBP modulate the correlations 
between SNAIL, ZEB1, E-cadherin and vitamin D receptor in human colon carcinomas. Int J Cancer. 2006;119(9):2098-104.

17. Peinado H, Olmeda D, Cano A. Snail, Zeb and bHLH factors in tumour progression: an alliance against the epithelial phenotype? Nat Rev Cancer. 2007;7(6):415-28.

18. Peinado H, Ballestar E, Esteller M, Cano A. Snail mediates E-cadherin repression by the recruitment of the Sin3A/histone deacetylase 1 (HDAC1)/HDAC2 complex. Mol Cell Biol. 2004;24(1):306-19.

19. Kendall J, Liu Q, Bakleh A, Krasnitz A, Nguyen KC, Lakshmi B, Gerald WL, Powers S, Mu D. Oncogenic cooperation and coamplification of developmental transcription factor genes in lung cancer. Proc Natl Acad Sci U S A. 2007;104(42):16663-8.

20. Cancer Genome Atlas Research N, Weinstein JN, Collisson EA, Mills GB, Shaw KR, Ozenberger BA, Ellrott K, Shmulevich I, Sander C, Stuart JM. The Cancer Genome Atlas Pan-Cancer analysis project. Nat Genet. 2013:45(10):1113-20.

21. West J, Bianconi G, Severini S, Teschendorff AE. Differential network entropy reveals cancer system hallmarks. Sci Rep. 2012;2:802.

22. Zhang HM, Chen H, Liu W, Liu H, Gong J, Wang H, Guo AY. AnimalTFDB: a comprehensive animal transcription factor database. Nucleic Acids Res. 2012;40(Database issue):D144-149.

23. Matys V, Kel-Margoulis OV, Fricke E, Liebich I, Land S, Barre-Dirrie A, Reuter I, Chekmenev D, Krull M, Hornischer K, et al. TRANSFAC and its module TRANSCompel: transcriptional gene regulation in eukaryotes. Nucleic Acids Res. 2006;34(Database issue):D108-110.

24. Fulton DL, Sundararajan $S$, Badis G, Hughes TR, Wasserman WW, Roach JC, Sladek R. TFCat: the curated catalog of mouse and human transcription factors. Genome Biol. 2009;10(3):R29.

25. Hammonds AS, Bristow CA, Fisher WW, Weiszmann R, Wu S, Hartenstein V, Kellis M, Yu B, Frise E, Celniker SE. Spatial expression of transcription factors in Drosophila embryonic organ development. Genome Biol. 2013;14(12):R140.

26. Wang Y, Zhang XS, Xia Y. Predicting eukaryotic transcriptional cooperativity by Bayesian network integration of genome-wide data. Nucleic Acids Res. 2009;37(18):5943-58.

27. Zhou XJ, Kao MC, Huang H, Wong A, Nunez-lglesias J, Primig M, Aparicio OM, Finch CE, Morgan TE, Wong WH. Functional annotation and network reconstruction through cross-platform integration of microarray data. Nat Biotechnol. 2005;23(2):238-43.

28. Patil A, Nakai K, Nakamura H. HitPredict: a database of quality assessed protein-protein interactions in nine species. Nucleic Acids Res. 2011; 39(Database issue):D744-749.

29. Consortium EP. An integrated encyclopedia of DNA elements in the human genome. Nature. 2012;489(7414):57-74.

30. Yu H, Liu BH, Ye ZQ, Li C, Li YX, Li YY. Link-based quantitative methods to identify differentially coexpressed genes and gene pairs. BMC bioinformatics. 2011;12:315.

31. Zheng CH, Yuan L, Sha W, Sun ZL. Gene differential coexpression analysis based on biweight correlation and maximum clique. BMC bioinformatics. 2014;15 Suppl 15:S3.

32. Hohmann AF, Vakoc CR. A rationale to target the SWI/SNF complex for cancer therapy. Trends Genet. 2014;30(8):356-63.

33. Washburn MP. There is no human interactome. Genome Biol. 2016;17(1):48.

34. Mar JC, Matigian NA, Mackay-Sim A, Mellick GD, Sue CM, Silburn PA, McGrath $\mathrm{JJ}$, Quackenbush J, Wells CA. Variance of gene expression identifies altered network constraints in neurological disease. PLoS Genet. 2011;7(8):e1002207.

35. Schaefer MH, Serrano L, Andrade-Navarro MA. Correcting for the study bias associated with protein-protein interaction measurements reveals differences between protein degree distributions from different cancer types. Front Genet. 2015;6:260.

36. Gillis J, Ballouz S, Pavlidis P. Bias tradeoffs in the creation and analysis of protein-protein interaction networks. J Proteomics. 2014;100:44-54.

37. Woo HG, Park ES, Lee JS, Lee YH, Ishikawa T, Kim YJ, Thorgeirsson SS. Identification of potential driver genes in human liver carcinoma by genomewide screening. Cancer Res. 2009;69(9):4059-66.

38. Yuan CX, Gurley WB. Potential targets for HSF1 within the preinitiation complex. Cell Stress Chaperones. 2000;5(3):229-42.

39. Lee SU, Maeda T. POKZZBTB proteins: an emerging family of proteins that regulate lymphoid development and function. Immunol Rev. 2012;247(1):107-19.

40. Acampora D, D'Esposito M, Faiella A, Pannese M, Migliaccio E, Morelli F, Stornaiuolo A, Nigro V, Simeone A, Boncinelli E. The human HOX gene family. Nucleic Acids Res. 1989;17(24):10385-402.
41. Savage Kl, Matchett KB, Barros EM, Cooper KM, Irwin GW, Gorski JJ, Orr KS, Vohhodina J, Kavanagh JN, Madden AF, et al. BRCA1 deficiency exacerbates estrogen-induced DNA damage and genomic instability. Cancer Res. 2014;74(10):2773-84.

42. Yanai $\mathrm{H}$, Negishi $\mathrm{H}$, Taniguchi T. The IRF family of transcription factors: Inception, impact and implications in oncogenesis. Oncoimmunology. 2012;1(8):1376-86.

43. Barnes B, Lubyova B, Pitha PM. On the role of IRF in host defense. J Interf Cytokine Res. 2002;22(1):59-71.

44. Mamane Y, Heylbroeck C, Genin P, Algarte M, Servant MJ, LePage C, DeLuca C, Kwon H, Lin R, Hiscott J. Interferon regulatory factors: the next generation. Gene. 1999;237(1):1-14.

45. Taniquchi T, Ogasawara K, Takaoka A, Tanaka N. IRF family of transcription factors as regulators of host defense. Annu Rev Immunol. 2001;19:623-55.

46. Bailey CM, Khalkhali-Ellis Z, Kondo S, Margaryan NV, Seftor RE, Wheaton WW Amir S, Pins MR, Schutte BC, Hendrix MJ. Mammary serine protease inhibitor (Maspin) binds directly to interferon regulatory factor 6: identification of a novel serpin partnership. J Biol Chem. 2005;280(40):34210-7.

47. Burchert A, Cai D, Hofbauer LC, Samuelsson MK, Slater EP, Duyster J, Ritter M, Hochhaus A, Muller R, Eilers M, et al. Interferon consensus sequence binding protein (ICSBP; IRF-8) antagonizes BCR/ABL and downregulates bcl-2. Blood. 2004:103(9):3480-9.

48. Mori T, Anazawa Y, liizumi M, Fukuda S, Nakamura Y, Arakawa H. Identification of the interferon regulatory factor 5 gene (IRF-5) as a direct target for p53. Oncogene. 2002;21(18):2914-8.

49. Yanai H, Chen HM, Inuzuka T, Kondo S, Mak TW, Takaoka A, Honda K, Taniguchi T. Role of IFN regulatory factor 5 transcription factor in antiviral immunity and tumor suppression. Proc Natl Acad Sci U S A. 2007;104(9):3402-7.

50. Barnes BJ, Kellum MJ, Pinder KE, Frisancho JA, Pitha PM. Interferon regulatory factor 5, a novel mediator of cell cycle arrest and cell death. Cancer Res. 2003;63(19):6424-31.

51. Takaoka A, Tamura T, Taniguchi T. Interferon regulatory factor family of transcription factors and regulation of oncogenesis. Cancer Sci. 2008;99(3):467-78

52. Futreal PA, Coin L, Marshall M, Down T, Hubbard T, Wooster R, Rahman N, Stratton MR. A census of human cancer genes. Nat Rev Cancer. 2004;4(3):177-83.

53. Davies H, Bignell GR, Cox C, Stephens P, Edkins S, Clegg S, Teaque J, Woffendin H, Garnett MJ, Bottomley W, et al. Mutations of the BRAF gene in human cancer. Nature. 2002;417(6892):949-54.

54. Abraham J, Stenger M. Dabrafenib in advanced melanoma with BRAF V600E mutation. J Community Support Oncol. 2014;12(2):48-9.

55. Stein S, Thomas EK, Herzog B, Westfall MD, Rocheleau JV, Jackson 2nd RS, Wang $M$, Liang P. NDRG1 is necessary for p53-dependent apoptosis. J Biol Chem. 2004;279(47):48930-40.

56. Zhang J, Chen S, Zhang W, Zhang J, Liu X, Shi H, Che H, Wang W, Li F, Yao L. Human differentiation-related gene NDRG1 is a Myc downstreamregulated gene that is repressed by Myc on the core promoter region. Gene. 2008:417(1-2):5-12.

57. Wang B, Li J, Ye Z, Li Z, Wu X. N-myc downstream regulated gene 1 acts as a tumor suppressor in ovarian cancer. Oncol Rep. 2014;31(5):2279-85.

58. Barrett T, Wilhite SE, Ledoux P, Evangelista C, Kim IF, Tomashevsky M, Marshall KA, Phillippy KH, Sherman PM, Holko M, et al. NCBI GEO: archive for functional genomics data sets-update. Nucleic Acids Res. 2013:41(Database issue):D991-995.

59. Huntley RP, Sawford T, Mutowo-Meullenet P, Shypitsyna A, Bonilla C, Martin MJ O'Donovan C. The GOA database: gene Ontology annotation updates for 2015. Nucleic Acids Res. 2015;43(Database issue):D1057-1063.

60. Subramanian A, Tamayo P, Mootha VK, Mukherjee S, Ebert BL, Gillette MA, Paulovich A, Pomeroy SL, Golub TR, Lander ES, et al. Gene set enrichment analysis: a knowledge-based approach for interpreting genome-wide expression profiles. Proc Natl Acad Sci U S A. 2005:102(43):15545-50.

61. Hollander M, Wolfe DA. Nonparametric statistical methods. New York: Wiley; 1973

62. Kel AE, Gossling E, Reuter I, Cheremushkin E, Kel-Margoulis OV, Wingender E. MATCH: A tool for searching transcription factor binding sites in DNA sequences. Nucleic Acids Res. 2003;31(13):3576-9.

63. Wang J, Duncan D, Shi Z, Zhang B. WEB-based GEne SeT AnaLysis Toolkit (WebGestalt): update 2013. Nucleic Acids Res. 2013;41(Web Server issue): W77-83. 